\title{
Influence of exogenous leptin on redox homeostasis in neutrophils and lymphocytes cultured in synovial fluid isolated from patients with rheumatoid arthritis
}

\author{
Michał Gajewski ${ }^{1}$, Joanna Gajewska², Przemysław Rzodkiewicz ${ }^{1,3}$, Elżbieta Wojtecka-Łukasik ${ }^{1}$ \\ ${ }^{1}$ Department of Biochemistry and Molecular Biology, National Institute of Geriatrics, Rheumatology and Rehabilitation, Warsaw, Poland \\ ${ }^{2}$ Screening Department, Institute of Mother and Child, Warsaw, Poland \\ ${ }^{3}$ Department of General and Experimental Pathology, II Faculty of Medicine, Medical University of Warsaw, Poland
}

\begin{abstract}
Objectives: Leptin is an adipose cells derived hormone that regulates energy homeostasis within the body. Energy metabolism of immune cells influences their activity within numerous pathological states, but the effect of leptin on these cells in unclear. On the one hand, it was observed that leptin induces neutrophils chemotaxis and modulates phagocytosis. On the other hand, neutrophils exposed to leptin did not display detectable $\mathrm{Ca}^{2+}$ ions mobilization or $\beta_{2}$-integrin upregulation. In this study, we investigated the effect of leptin on the redox homeostasis in lymphocytes and neutrophils. Material and methods: Neutrophils and lymphocytes were isolated by density-gradient centrifugation of blood from healthy volunteers. Cells were cultured with or without leptin $(100 \mathrm{ng} / \mathrm{ml}$ for lymphocytes and $500 \mathrm{ng} / \mathrm{ml}$ for neutrophils) or with or without synovial fluid (85\%) for 0-72 h. Culture media were not changed during incubation. Cells were homogenized and homogenate was frozen until laboratory measurements. Redox homeostasis was assessed by the reduced glutathione (GSH) vs. oxidized glutathione (GSSG) ratio and membrane lipid peroxidation evaluation.

Results: Lymphocytes cultured with leptin and synovial fluid showed a significant increase of the GSSG level. The GSSG/GSH ratio increased by $184 \pm 37 \%$. In neutrophils incubated in a similar environment, the GSSG/GSH ratio increased by just $21 \pm 7 \%$, and the effect was observed irrespectively of whether they were exposed to leptin or synovial fluid or both together. Neither leptin nor synovial fluid influenced lipid peroxidation in neutrophils, but in lymphocytes leptin intensified lipid peroxidation. Conclusions: Leptin altered the lymphocytes, but not neutrophils redox state. Because firstly neutrophils are anaerobic cells and have just a few mitochondria and secondly lymphocytes have typical aerobic metabolism, the divergence of our data supports the hypothesis that leptin induces oxidative stress by modulation of mitochondria.
\end{abstract}

Key words: leukocytes, lipid peroxidation, leptin, glutathione.

\section{Introduction}

Leptin modulates the development, proliferation, maturation, activation and apoptosis of immune cells [1]. So far, leptin receptors have been found in neutrophils, monocytes, and lymphocytes [1]. The influence of leptin on neutrophils is unclear. On the one hand, leptin promotes neutrophils' chemotaxis and modu- lates neutrophils' phagocytosis of bacteria [2]. In diabetic patients' neutrophils, it was observed that an increase in serum leptin levels correlates with the degree of CD11b expression on neutrophils [3]. On the other hand, neutrophils exposed to leptin did not display detectable $\mathrm{Ca}^{2+}$ ions mobilization or $\beta_{2}$-integrin upregulation [4]. 
Previous studies on the influence of leptin on neutrophils' oxidative activity bring contradictory results. Caldefie-Chezet et al. [5] detected a significant increase in oxidant production by leptin in human neutrophils, but, in contrast, Ottonello et al. [2] noted that neutrophils exposed to leptin did not display oxidant production.

In our previous study [6], we found that leptin does not affect the level of the chemiluminescence $(\mathrm{CL})$ in inactive neutrophils incubated in normal serum, and it does not modulate the level of oxidative activity in resting neutrophils incubated with synovial fluid (SF). However, leptin reduces emission of extracellular reactive oxygen intermediates (ROI) in activated neutrophils, and that effect is dependent on the concentration and duration of exposure to leptin. The role of leptin in modulation of the oxidative activity of neutrophils is still an open question. The influence of leptin on the metabolism of neutrophils infiltrating the joints of patients with rheumatoid arthritis (RA) should be carefully examined. In another study of ours, we suggested that neutrophils' oxidative activity should be investigated in an experimental model simulating conditions similar to those prevailing in the joint - physiological concentrations of SF [7].

The aim of this study was to analyze the oxidative/ antioxidative status in neutrophils and lymphocytes cultured in SF from patients with RA and to correlate obtained redox markers with leptin level. Redox homeostasis was evaluated by the reduced to oxidized glutathione ratio (GSH/GSSG) and lipid peroxidation (LPO) level. The measurement was performed in homogenates from immune cells: neutrophils and lymphocytes cultured in physiological concentrations of SF.

\section{Material and methods}

\section{Chemicals}

Leptin was obtained from PeproTech. Phosphate buffered saline (PBS) and Gradisol $G$ and $L$ were obtained from Polfa, Poland. The cells were cultured in DMEM from PAA Lab GmbH, Austria. Human (GSH) Elisa Kit and Human (GSSG) Elisa Kit were obtained from DRG Instruments $\mathrm{GmbH}$, Germany.

\section{Preparation of synovial fluid}

Synovial fluid samples were obtained from patients with RA, centrifuged at 10,000 g for 30 minutes, divided into $1 \mathrm{ml}$ aliquots and stored at $-20^{\circ} \mathrm{C}[6]$.

\section{Leukocyte isolation}

Neutrophils and lymphocytes were isolated by density-gradient centrifugation, from heparinized blood of healthy volunteers by Gradisol G ( $d=1.119 \mathrm{~g} / \mathrm{cm}^{3}$ - neu- trophils) and Gradisol L ( $\mathrm{d}=1.077 \mathrm{~g} / \mathrm{cm}^{3}$ - lymphocytes) after hypotonic shock lysis of contaminating red blood cells. Neutrophils and lymphocytes were morphologically normal, and their viability was $>90 \%$ (trypan blue exclusion test). Cells were used within 3 hours of preparation.

\section{Biochemical assays}

Cell cultures with or without leptin $(100 \mathrm{ng} / \mathrm{ml}$ for lymphocytes and $500 \mathrm{ng} / \mathrm{ml}$ for neutrophils) were incubated for 0-72 h. Culture media were not changed during incubation. After the experiment, media were removed after centrifugation. The cell pellet was homogenized and homogenate was frozen until laboratory measurements.

To determine the level of lipid peroxidation, malondialdehyde (MDA) was assayed as in [8] by measuring the amount of thiobarbituric acid (TBA)-reactive materials. Intracellular reduced (GSH) and oxidized (GSSG) glutathione content was quantified according to the manufacturers' protocol.

\section{Results}

In our preliminary experiments, we tested the influence of leptin concentration on redox homeostasis (GSH/GSSG balance) and lipid peroxidation (LPO) of lymphocytes and neutrophils cell membranes. The minimal recordable effect of leptin on GSSG/GSH ratio in lymphocytes was observed at a concentration of $50 \mathrm{ng} / \mathrm{ml}$, whereas a ten times higher level was required to indicate a response from neutrophils (data not shown). Therefore, for the further experiments, we chose $100 \mathrm{ng} / \mathrm{ml}$ and $500 \mathrm{ng} / \mathrm{ml}$ concentrations of leptin for lymphocytes and neutrophils respectively.

After 24 hours of incubation, we observed a slight but constant increase of GSSG/GSH levels resulting in redox imbalance. The effect was observed irrespectively of whether it was exposure to leptin or SF or together (Fig. 1). This effect is probably related to maturation and apoptosis of neutrophils, which are short living cells (confirmed by observations after staining cells with trypan blue - data not shown).

After $24 \mathrm{~h}$ of incubation, we observed a significant increase in GSSG level. The GSSG/GSH ratio increased by $184 \pm 37 \%$. After the first day of the experiment we found a small decrease of this ratio, but even after 72 hours it was much higher than the output level (Fig. 2). It seems that leptin increase redox imbalance in lymphocytes but with increasing time of exposure the effect is weaker.

Neither leptin nor SF influenced lipid peroxidation in neutrophils, but in lymphocytes leptin intensified LPO (Figs. 3 and 4). An interesting synergistic effect was observed on lymphocytes incubated with both SF and leptin, because SF itself did not modulate LPO detectably (Fig. 4). 


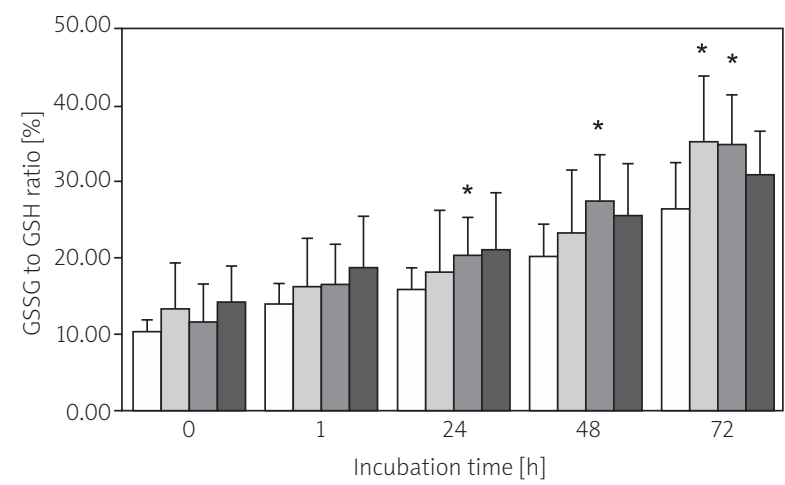

$\square \mathrm{N} \quad \square \mathrm{N}+\mathrm{SF} 85 \% \quad \square \mathrm{N}+$ Leptin $\quad \square \mathrm{N}+$ Leptin + SF85\%

N-neutrophils; SF85\% - synovial fluid 85\%. Differences between groups were tested with Mann-Whitney $U$ test with a level of significance $\alpha=0.05$. * - compared to $N$ group.

Fig. 1. GSSG to GSH ratio in neutrophils incubated with $500 \mathrm{ng} / \mathrm{ml}$ leptin. Data are presented as mean \pm SD for 12 patients.

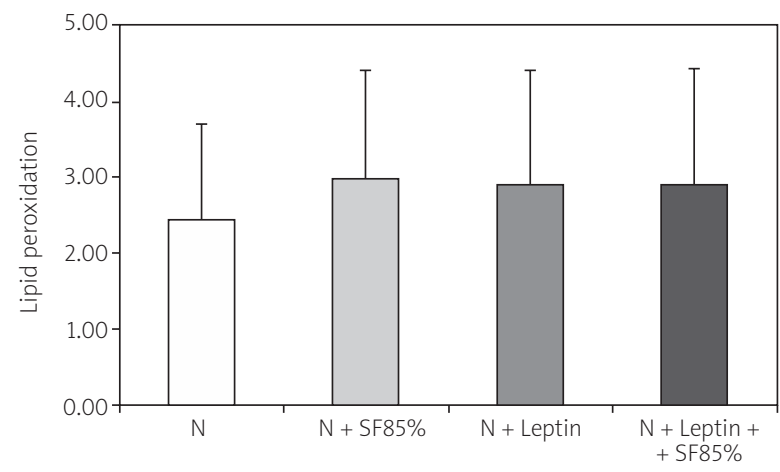

$N$ - control neutrophils cells, DMEM for $24 \mathrm{~h}, \mathrm{~N}+$ SF85\% - neutrophils cultured in $85 \%$ synovial fluid (SF) for $24 h ; N+$ leptin - neutrophils treated by $500 \mathrm{ng} / \mathrm{ml}$ leptin for $24 \mathrm{~h}$; N + leptin + SF85\% - neutrophils treated by $500 \mathrm{ng} / \mathrm{ml}$ leptin and cultured for $24 \mathrm{~h}$ in SF85\%. Differences between groups were tested with Mann-Whitney $U$ test with a level of significance $\alpha=0.05$.

Fig. 3. Effect of leptin on the lipid peroxidation (LPO) level in neutrophils (ng/mg protein).

\section{Discussion}

Leptin acts on energy metabolism and plays a role in the modulation of cellular redox balance, but the latest research on the regulation of oxidative metabolism of various cells by leptin has brought contradictory data. Caldefie-Chezet et al. [5] showed for the first time that leptin may influence redox homeostasis. The researchers detected the leptin receptor on neutrophils and demonstrated that leptin increases ROI production in stimulated neutrophils [5]. These observations were confirmed by Fernandez-Riejos et al. [1], who showed that leptin is an essential activator of the immune sys-

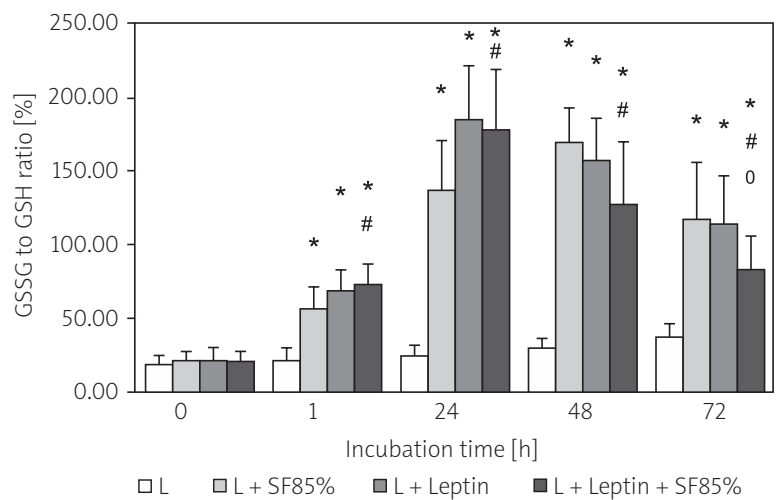

L - lymphocytes; SF85\% - synovial fluid 85\%. Differences between groups were tested with Mann-Whitney $U$ test with a level of significance $\alpha=0.05$ * - compared to N group; \# - compared to L + SF $85 \%$ group; o - compared to $L+$ Leptin group.

Fig. 2. GSSG to GSH ratio in lymphocytes incubated with $100 \mathrm{ng} / \mathrm{ml}$ leptin. Data are presented as mean \pm SD for 14 patients.

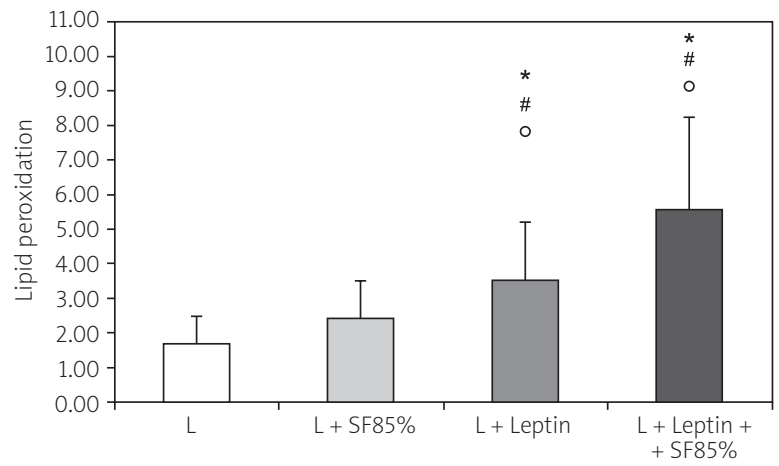

L - control lymphocytes cells. DMEM for $24 h . L+$ SF85\% - lymphocytes cultured in $85 \%$ synovial fluid (SF) for $24 \mathrm{~h}, \mathrm{~L}+$ leptin - lymphocytes treated by $100 \mathrm{ng} / \mathrm{ml}$ leptin for $24 \mathrm{~h}$; L leptin + SF $85 \%$-lymphocytes treated by $100 \mathrm{ng} / \mathrm{ml}$ leptin and cultured in SF85\% for $24 \mathrm{~h}$. Differences between groups were tested with Mann-Whitney U test with a level of significance $\alpha=0.05 ;{ }^{*}$ - compared to N group; \# - compared to L + SF $85 \%$ group; 0 - compared to $L+$ Leptin group

Fig. 4. Effect of leptin on lipid peroxidation (LPO) level in human lymphocytes/monocytes (ng/mg protein).

tem and inflammatory mediator that affects neutrophils and other immunological cells. Opposite findings were presented by Kamp et al. [9], who observed that leptin in a physiological concentration $(250 \mathrm{ng} / \mathrm{ml})$ does not influence on neutrophils at all. Only in extremely high concentrations (25000 ng/ml) does leptin induce some changes in the proteome of neutrophils, but this effect is most likely mediated by indirect effect consisting of a protective action on acceleration, activation and cell survival [9]. Moreover, there was no influence on polymorphonuclear leukocyte chemotaxis [9]. Recently there has been no clear consensus on leptin's influence on neutrophils. 
It is commonly known that ROI have an impact on oxidative status and proliferation of various cells. It is believed that leptin might also regulate these processes, but the type of this action is unclear. Some reports have suggested that leptin may increase antioxidant enzymes activity by regulation of membrane lipids peroxidation (LPO). In the acute response to renal ischemia-reperfusion in rats, it was observed that leptin decreases the level of the oxidative stress marker MDA and increases the activity of antioxidant enzyme scavengers [10]. Equivalent results were obtained in studies by Zwirska-Korczala et al. [11] and Schling and Loffler [12], who observed that leptin decreased MDA levels in a dose-dependent manner in an in vitro study of preadipocyte cells. Zheng at al. [13] demonstrated that leptin abolishes augmented LPO in cardiomyocytes by restoring the activity of antioxidant enzymes. Contrary to that, Bouloumie et al. [14] found that in cultured endothelial cells leptin-induced $\mathrm{ROI}$ production. It was suggested that leptin increases mitochondrial electron transport chain activity and in turn increases mitochondrial production of ROI [14]. Similar results were obtained by Kutlu et al. [15], who observed that leptin increases peroxidation of lipids in the mouse brain. Alteration of the cellular redox state was also observed by Savini et al. [16] who found that in keratinocytes cultured with supplementation of leptin an increased GSH/GSSG ratio was observed. This effect was blocked by inhibitors of electron transport and uncouplers of oxidative phosphorylation. These data suggested that leptin induces adaptation to oxidative stress by modulation of mitochondrial homeostasis [16]. Leptin seems to exert a different effect on different cells, sometimes increasing, sometimes decreasing oxidative status.

In a recent study, we examined the influence of leptin on oxidative metabolism of neutrophils and other leukocytes in physiological concentrations of SF. Our study, on the one hand, showed that neither leptin nor synovial fluid influences the GSSG/GSH ratio or lipid peroxidation in neutrophils. On the other hand, we observed that both SF and leptin increase redox imbalance, and we observed a synergistic effect between SF and leptin on the LPO level in lymphocytes. Thus we found a differential effect of leptin on various immune cells. Similar results were obtained in our previous study, where SF did not have any impact on glutathione homeostasis but, depending on the concentration of leptin, increased extracellular and decreased general $\mathrm{ROI}$ production by neutrophils [6]. This observation suggests that small changes in neutrophils are purely related to a mechanical shift. Growth in lipid peroxidation in lymphocytes confirms that not the physical (pressure) but the biochemical mechanism is responsible for the redox imbalance in these cells.
In neutrophils energy metabolism is limited to glucose. The few mitochondria that are present in the cells play a role in apoptosis only. In contrast to neutrophils, lymphocytes in a resting state primarily uses mitochondria to derive energy from the process of oxidative phosphorylation [17, 18]. Only after activation in the course of inflammation do they switch their metabolism to anaerobic glycolysis. This phenomenon is related to reduced oxygen concentration in inflamed tissue and is essential for several immunological functions of these cells such as clonal expansion and the production of antibodies and cytokines [19, 20].

Resting lymphocytes produce $88 \%$ of ATP in the process of oxidative phosphorylation while activated or proliferating cells produce $86 \%$ from anaerobic glycolysis [17]. ROI are a byproduct of the energy-generating process in mitochondria. The intensity of oxidative stress in activated lymphocytes is related to oxidative phosphorylation process. Minimization of oxidative stress, by decreasing ROI production during cellular activation, is a cell-protecting strategy, but the result is their longer life at the expense of reduced ability to regulate the inflammatory process [18].

\section{Conclusion}

Because neutrophils are anaerobic cells and have just a few mitochondria and lymphocytes have typical aerobic metabolism, the divergence of our data supports the hypothesis that leptin induces oxidative stress by modulation of mitochondria.

Immune cells differentially regulate redox balance depending on their metabolism [20, 21]. In neutrophils, anaerobic metabolism dominates, while in lymphocytes and monocytes aerobic metabolism shifts to anaerobic glycolysis in the course of inflammation. The possibility of influencing those processes may be an alternative method of treatment of inflammatory diseases.

The authors declare no conflict of interest.

\section{References}

1. Fernandez-Riejos P, Najib S, Santos-Alvarez J. Role of leptin in the activation of immune cells. Mediators Inflamm 2010; 2010: 568343; doi: 10.1155/2010/568343.

2. Ottonello L, Gnerre P, Bertolotto $M$, et al. Leptin as a uremic toxin interferes with neutrophil chemotaxis. J Am Soc Nephrol 2004; 15: 2366-2372.

3. Mastej K, Adamiec R. Neutrophil surface expression of adhesion molecule CD11b in patients with type 2 diabetes. Przegl Lek 2009; 66: 228-232.

4. Montecucco F, Bianchi G, Gnerre P, et al. Induction of neutrophil chemotaxis by leptin: crucial role for p38 and Src kinases. Ann N Y Acad Sci 2006; 1069: 463-471. 
5. Caldefie-Chezet F, Poulin A, Tridon A, et al. Leptin: a potential regulator of polymorphonuclear bactericidal action? J Leukoc Biol 2001; 69: 414-418.

6. Gajewski M, Rzodkiewicz P, Gajewska J, Wojtecka-Łukasik E. The effect of leptin on the respiratory burst of human neutrophils cultured in synovial fluid. Reumatologia 2015; 53: 21-25.

7. Gajewski M, Szczepanik S, Wysocki $t$, et al. the effect of increasing amounts of synovial fluid isolated from patients with rheuamtoid arthritis on the respiratory burst of human neutrophils. Reumatologia 2009; 47: 273-281.

8. Kramer K, Rademaker B, Rozendal WH, et al. Influence of lipid peroxidation on B-adrenoceptors. FEBS Lett 1986; 198: 80-86.

9. Kamp VM, Langereis JD, Aalst van CW, et al. Physiological concentrations of leptin do no affect human neutrophils. PLoS ONE 2013; 8: e73170; doi: 10.1371/journal.pone.0073170.

10. Erkasap S, Erkasap N, Koken T, et al. Effect of leptin on renal ischemia-reperfusion damage in rats. J Physiol Biochem 2004; 60: 79-84.

11. Zwirska-Korczala K, Adamczyk-Sowa M, Sowa P, et al. Role of leptin, gherelin, angiotensin II and orexins in $3 \mathrm{~T} 3 \mathrm{~L}$ 1 preadipocyte cells proliferation and oxidative metabolism. J Physiol Pharmacol 2007; 58 (suppl. 1): 53-64.

12. Schling P, Loffler G. Effects of angiotensin II on adipose conversion and expression of genesof the renin-angiotensin system in human preadipocytes. Hormon Metab Res 2001; 33: 189-195.

13. Zheng J, Fang J, Yin YJ, et al. Leptin protects cardiomyocytes from serum-deprivation-induced apoptosis by increasing anti-oxidant defence. Clin Exp Pharmacol Physiol 2010; 37: 955-962.

14. Bouloumie A, Marumo T, Lafontan M, Busse R. Leptin induces oxidative stress in human endothelial cells. FASEB J 1999; 13: 1231-1238.

15. Kutlu S, Canpolat S, Aydin M, et al. Exogenous leptin increases lipid peroxidation in the mouse brain. Tohoku J Exp Med 2005; 206: 233-236.

16. Savini I, Catani MV, Rossi A, et al. Vitamin C recycling is enhanced in the adaptive response to leptin-induced oxidative stress in keratinocytes. J Invest Dermatol 2003; 121: 786-793.

17. Brand KA, Hermfisse U. Aerobic glycolysis by proliferating cells: a protective strategy against oxygen species. FASEB J 1997; 11: 388-395.

18. Kramer PA, Ravi S, Chacko B, et al. A review of the mitochondrial and glycolytic metabolism in human platelets and leukocytes: Implications for their use as bioenergetic biomarkers. Redox Biol 2014; 2: 206-210.

19. Krauss S, Brand MD, Buttgereit F. Signaling takes a breathnew quantitative perspectives on bioenergetics and signal transduction, Immunity 2001; 15: 497-502.

20. Pearce EL, Poffenberger MC, Chang CH, Jones RG. Fueling immunity: insights into metabolism and lymphocyte function. Science 2013; 342 (6155): 1242454.

21. Gajewski M, Rzodkiewicz P, Wojtecka-Łukasik E. The role of physiological elements in the future therapies of rheumatoid arthritis. II. The relevance of energy redistribution in the process of chronic inflammation. Reumatologia 2015; 53: 1-6. 